\title{
Contribution of Water to Pressure and Cold Denaturation of Proteins
}

\author{
Valentino Bianco and Giancarlo Franzese \\ Departament de Física Fonamental, Universitat de Barcelona, Martí i Franquès 1, 08028 Barcelona, Spain \\ (Received 11 May 2015; revised manuscript received 14 July 2015; published 1 September 2015)
}

\begin{abstract}
The mechanisms of cold and pressure denaturation of proteins are matter of debate and are commonly understood as due to water-mediated interactions. Here, we study several cases of proteins, with or without a unique native state, with or without hydrophilic residues, by means of a coarse-grain protein model in explicit solvent. We show, using Monte Carlo simulations, that taking into account how water at the protein interface changes its hydrogen bond properties and its density fluctuations is enough to predict protein stability regions with elliptic shapes in the temperature-pressure plane, consistent with previous theories. Our results clearly identify the different mechanisms with which water participates to denaturation and open the perspective to develop advanced computational design tools for protein engineering.
\end{abstract}

DOI: 10.1103/PhysRevLett.115.108101

PACS numbers: 87.15.Cc, 87.15.A-, 87.15.kr

Water plays an essential role in driving the folding of a protein and in stabilizing the tertiary protein structure in its native state $[1,2]$. Proteins can denaturate-unfolding their structure and losing their activity - as a consequence of changes in the environmental conditions. Experimental data show that for many proteins the native folded state is stable in a limited range of temperatures $T$ and pressures $P$ [3-8] and that partial folding is $T$ modulated also in "intrinsically disordered proteins" [9]. By hypothesizing that proteins have only two different states, folded $(f)$ and unfolded $(u)$, and that the $f \longleftrightarrow u$ process is reversible at any moment, Hawley proposed a theory [10] that predicts a close stability region (SR) with an elliptic shape in the $T-P$ plane, consistent with the experimental data [11].

Cold and $P$ denaturation of proteins have been related to the equilibrium properties of the hydration water [12-23]. However, the interpretations of the mechanism are still controversial [8,24-37]. High- $T$ denaturation is easily understood in terms of thermal fluctuations that disrupt the compact protein conformation: the open protein structure increases the entropy $S$ minimizing the global Gibbs free energy $G \equiv H-T S$, where $H$ is the total enthalpy. High- $P$ unfolding can be explained by the loss of internal cavities in the folded states of proteins [36], while denaturation at negative $P$ has been experimentally observed [38] and simulated $[38,39]$ recently. Cold and $P$ unfolding can be thermodynamically justified assuming an enthalpic gain of the solvent upon the denaturation process, without specifying the origin of this gain from molecular interactions [40]. Here, we propose a molecular-interactions model for proteins solvated by explicit water, based on the "many-body" water model [32,41-45]. We demonstrate how the cold- and $P$-denaturation mechanisms can emerge as a competition between different free energy contributions coming from water, one from hydration water and another from bulk water. Moreover, we show how changes in the protein sequence affect the hydration water properties and, in turn, the stability of the protein folded staterelevant information in protein design [27].

The many-body water model adopts a coarse-grain (CG) representation of the water coordinates by partitioning the available volume $V$ into a fixed number $N_{0}$ of cells, each with volume $v \equiv V / N_{0} \geq v_{0}$, where $v_{0}$ is the water excluded volume. Each cell accommodates at most one molecule with the average $\mathrm{O}-\mathrm{O}$ distance between nearest neighbor (NN) water molecules given by $r=v^{1 / 3}$. To each cell we associate a variable $n_{i}=1$ if the cell $i$ is occupied by a water molecule and has $v_{0} / v>0.5$, and $n_{i}=0$ otherwise. Hence, $n_{i}$ is a discretized density field replacing the water translational degrees of freedom. The Hamiltonian of the bulk water

$$
\mathcal{H} \equiv \sum_{i j} U\left(r_{i j}\right)-J N_{\mathrm{HB}}^{(b)}-J_{\sigma} N_{\text {coop }}
$$

has a first term, summed over all the water molecules $i$ and $j$ at $\mathrm{O}-\mathrm{O}$ distance $r_{i j}$, accounting for the van der Waals interaction, with $U(r) \equiv \infty$ for $r<r_{0} \equiv v_{0}^{1 / 3}=2.9 \AA$ (water van der Waals diameter), $U(r) \equiv 4 \epsilon\left[\left(r_{0} / r\right)^{12}-\right.$ $\left.\left(r_{0} / r\right)^{6}\right]$ for $r \geq r_{0}$ with $\epsilon \equiv 5.8 \mathrm{~kJ} / \mathrm{mol}$, and $U(r) \equiv 0$ for $r>r_{c} \equiv 6 r_{0}$ (cutoff).

The second term represents the directional component of the hydrogen bond (HB), with $J / 4 \epsilon=0.3[46], N_{\mathrm{HB}}^{(b)} \equiv$ $\sum_{\langle i j\rangle} n_{i} n_{j} \delta_{\sigma_{i j}, \sigma_{j i}}$ number of bulk HBs, with the sum over $\mathrm{NN}$, where $\sigma_{i j}=1, \ldots, q$ is the bonding index of molecule $i$ to the NN molecule $j$, with $\delta_{a b}=1$ if $a=b, 0$ otherwise. Each water molecule can form up to four HBs that break if $n_{i} n_{j}=0$, i.e., $r_{i j}>2^{1 / 3} r_{0}=3.6 \AA$ or $\widehat{\mathrm{OOH}}>30^{\circ}$. Hence, only $1 / 6$ of the entire range of values $\left[0,360^{\circ}\right]$ for the $\widehat{\mathrm{OOH}}$ angle is associated to a bonded state. Thus, we choose $q=$ 6 to account correctly for the entropy variation due to HB formation and breaking.

The third term, with $N_{\text {coop }} \equiv \sum_{i} n_{i} \sum_{(l, k)_{i}} \delta_{\sigma_{i k}, \sigma_{i l}}$, where $(l, k)_{i}$ indicates each of the six different pairs of the four 
indices $\sigma_{i j}$ of a molecule $i$, accounts for the HB cooperativity due to the quantum many-body interaction [47] and leads to the low- $P$ tetrahedral structure [48]. We choose $J_{\sigma} / 4 \epsilon \equiv 0.05 \ll J$ to guarantee an asymmetry between the two HB terms.

Increasing $P$ partially disrupts the open structure of the HB network and reduces $v$ toward $v_{0}$. We account for this with an average enthalpy increase $P v_{\mathrm{HB}}^{(b)}$ per $\mathrm{HB}$, where $v_{\mathrm{HB}}^{(b)} / v_{0}=0.5$ is the average volume increase between high- $\rho$ ices VI and VIII and low- $\rho$ (tetrahedral) ice Ih. Hence, the total bulk volume is $V^{(b)} \equiv N v_{0}+N_{\mathrm{HB}}^{(b)} v_{\mathrm{HB}}^{(b)}$. We assume that the HBs do not affect the NN distance $r$, consistent with experiments [48], and, hence, do not affect the $U(r)$ term.

Next we account for the effects of protein-water interaction. Experiments and simulations show that near a hydrophobic $(\Phi)$ residue water-water HBs are more stable than in bulk [15,49-51] with stronger water-water correlation [52]. We model this by replacing $J$ of Eq. (1) with $J_{\Phi}>J$ for HBs at the $\Phi$ interface. This choice, according to Muller [40], ensures the water enthalpy compensation during the cold denaturation [53].

The interaction at the $\Phi$ interface affects the hydration water density and fluctuations. Some works suggest a decrease of interfacial water density [54-57], while recent simulations show an increase of density in the first hydration shell of any solute [58] and an increase of compressibility near $\Phi$ solutes with size $\gtrsim 0.5 \mathrm{~nm}$ for water $[29,52,59]$ or waterlike solvents [60] with respect to bulk. Increasing $P$ induces a further increase of density and reduces the effect of the $\Phi$ interface on the compressibility of the hydration shell $[29,52,61,62]$. We incorporate this behavior into the model by using the following considerations. From the equilibrium condition for the thermodynamic potential of hydration water and the coexisting vapor at the $\Phi$ interface at fixed $T$, according to Eq. (2) of Ref. [63], we deduce $v^{(\Phi)}-v_{0} \sim\left(P-P^{*}\right)^{-1}$, where $v^{(\Phi)}$ is the volume per $\Phi$ hydration water molecule and $P^{*}<0$ is the equilibrium vapor pressure at the given $T$. If we attribute this $P$ dependence to the interfacial HB properties $\left[v_{\mathrm{HB}}^{(\Phi)} \sim v^{(\Phi)}-\right.$ $v_{0}$ ] and expand it as a power series in $P$, the average volume change per water-water $\mathrm{HB}$ at the $\Phi$ interface is

$$
v_{\mathrm{HB}}^{(\Phi)} / v_{\mathrm{HB}, 0}^{(\Phi)} \equiv 1-k_{1} P+k_{2} P^{2}-k_{3} P^{3}+O\left(P^{4}\right),
$$

where $v_{\mathrm{HB}, 0}^{(\Phi)}$ is the volume change associated to the $\mathrm{HB}$ formation in the $\Phi$ hydration shell at $P=0, k_{i}>0 \forall i$, and $\lim _{P \rightarrow \infty} v_{\mathrm{HB}}^{(\Phi)}=0$. Hence, the total volume $V$ is

$$
V \equiv V^{(b)}+V^{(\Phi)} \equiv V^{(b)}+N_{\mathrm{HB}}^{(\Phi)} v_{\mathrm{HB}}^{(\Phi)},
$$

where $V^{(\Phi)}$ and $N_{\mathrm{HB}}^{(\Phi)}$ are the $\Phi$ hydration shell volume and number of HBs, respectively.

Because we are interested in small values of $P$, i.e., near the biologically relevant atmospheric pressure, we include in our calculations only the linear term in Eq. (2) [64]. In the following we fix $k_{1}=1 v_{0} / 4 \epsilon, v_{\mathrm{HB}, 0}^{(\Phi)} / v_{0}=v_{\mathrm{HB}}^{(b)} / v_{0}=$ 0.5 , and $J_{\Phi} / J=1.83$. Our results have minor qualitative differences by including up to the third order in Eq. (2) or by changing the parameters up to $50 \%$.

Because our goal here is to calculate the water contribution to denaturation, we model the protein as a selfavoiding $\Phi$ homopolymer, without internal cavities [65], whose residues occupy NN cells with no residue-residue interaction but the excluded volume, as in other CG approaches to the problem $[13-15,66]$. This implies that the protein has several "native" states, all with the same maximum number $n_{\max }$ of residue-residue contacts. To simplify the discussion, we initially neglect energetic contributions of the water- $\Phi$ residue interaction.

We analyze the system by Monte Carlo simulations at constant $N, P$, and $T$. We adopt a representation in two dimensions [12-15,67], using a square partition, to favor visualization and understanding of our results. Comparisons with our preliminary results in three dimensions do not show qualitative changes, mainly because the number of $\mathrm{NN}$ water molecules is four both in two dimensions and three dimensions for the tendency of water to form tetrahedral structures in three dimensions.

We consider that the protein is folded if the average number of residue-residue contacts $n_{\mathrm{rr}} \geq 50 \% n_{\max }$. We find an elliptic SR (Fig. 1), consistent with experiments and the Hawley theory $[10,11]$, with heat, cold, and $P$ unfolding. The elliptic shape is preserved when we change the threshold for $n_{\mathrm{rr}}$, showing that the $f \longleftrightarrow u$ is a continuous process. In the SR the folded protein [Fig. 2(a)] minimizes the number of hydrated $\Phi$ residues, reducing the energy cost of the interface, as expected.

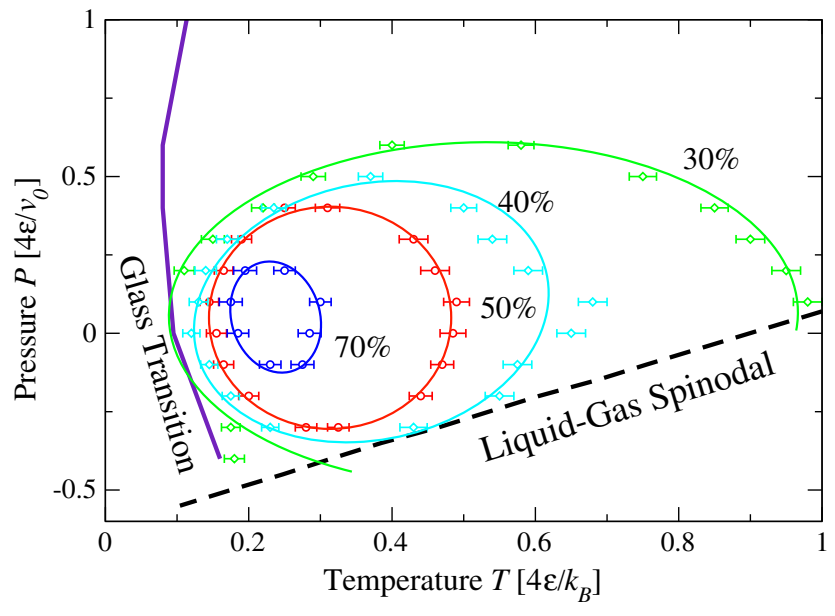

FIG. 1 (color online). $\quad P-T$ SR of the protein from Monte Carlo simulations. Symbols mark state points with the same average residue-residue contact's number $n_{\mathrm{rr}} / n_{\max }=30 \%, 40 \%, 50 \%$ and $70 \%$. Elliptic lines are guides for the eyes. The "glass transition" line defines the temperatures below which the system does not equilibrate. The spinodal line marks the stability limit of the liquid phase at high $P$ with respect to the gas at low $P ; k_{B}$ is the Boltzmann constant. 

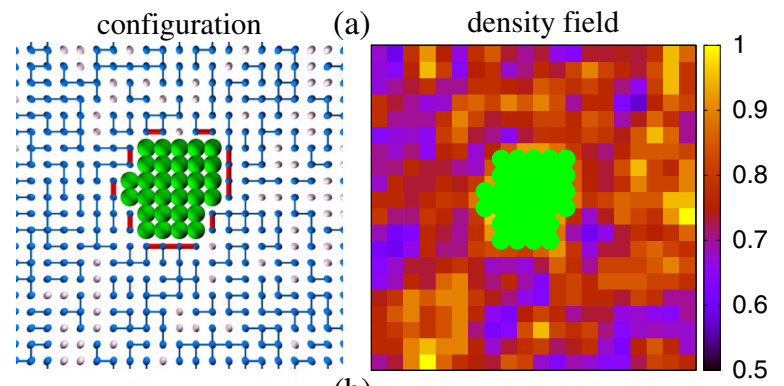

(b)

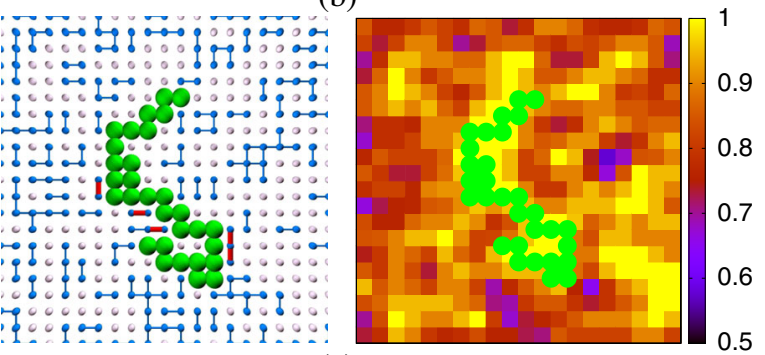

(c)

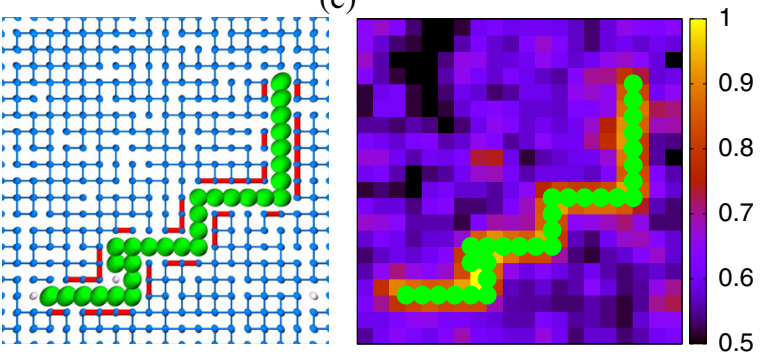

(d)

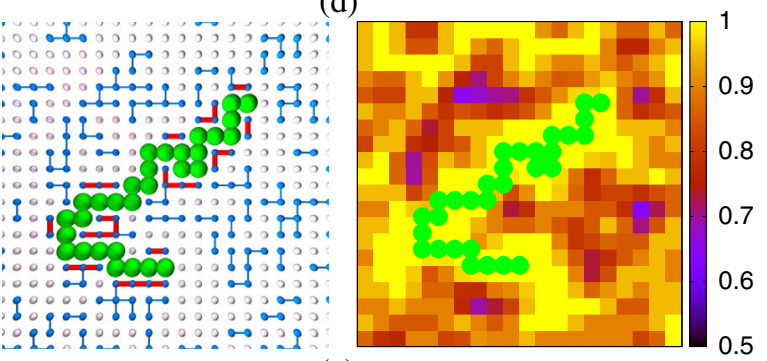

(e)
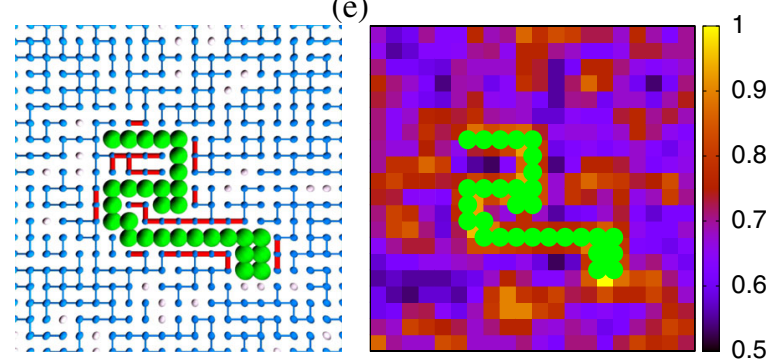

FIG. 2 (color online). Typical configurations of a hydrated protein made of 30 residues (in green): (a) folded at the state point $\left(T k_{B} / 4 \epsilon, P v_{0} / 4 \epsilon\right)=(0.25,0.1)$ and unfolded (b) at high $T$ $(0.9,0.1),(\mathrm{c})$ at low $T(0.1,0.1),(\mathrm{d})$ at high $P(0.25,0.6),(\mathrm{e})$ at low $P(0.25,-0.3)$. Left panels: Water molecules with (without) HBs are represented in blue (white) and bulk (interfacial) HBs in blue (red). Right panels: Color coded water density field (from black for lower $\rho$ to yellow for higher $\rho$ ) calculated as $v_{0} \rho_{i}^{(\lambda)} \equiv v_{0} /\left(v_{0}+n_{\mathrm{HB}, i}^{(\lambda)} v_{\mathrm{HB}}^{(\lambda)}\right)$ where $\lambda=b, \Phi$, and $n_{\mathrm{HB}, i}^{(\lambda)}$ is the number of HBs associated to the water molecule $i$, with $\sum_{i} n_{\mathrm{HB}, i}^{(\lambda)}=N_{\mathrm{HB}}^{(\lambda)}$.
First, we observe that the model reproduces the expected entropy-driven $f \longleftrightarrow u$ for increasing $T$ at constant $P$ [Fig. 2(b)]. The entropy $S$ increases both for the opening of the protein and for the larger decrease of HBs.

Upon isobaric decrease of $T$ the internal energy dominates the system Gibbs free energy [Fig. 2(c)]. However, $N_{\mathrm{HB}}^{(b)}$ saturates at $T$ lower than the SR; therefore, the only way for the system to further minimize the internal energy is to increase $N_{\mathrm{HB}}^{(\Phi)}$, i.e., to unfold the protein. Hence, the cold denaturation is an energy-driven process toward a protein state that is stabilized by the increased number of HBs in the hydration shell.

Upon isothermal increase of $P$, the protein denaturates [Fig. 2(d)]. We find that this change is associated to a decrease of $N_{\mathrm{HB}}^{(b)}$ and a small increase of $N_{\mathrm{HB}}^{(\Phi)}$ that lead to a net decrease of $V$ at high $P$, as a consequence of Eqs. (2) and (3), and an increase of internal energy. At high $P$ the $P V$ decrease associated to the $f \rightarrow u$ process at constant $T$ dominates over the concomitant internal energy increase, determining a lower Gibbs free energy for the $u$ state. Hence, water contribution to the high- $P$ denaturation is density driven, as emphasized by the increase of local density near the unfolded protein.

Finally, upon isothermal decrease of $P$ toward negative values [Fig. 2(e)], the enthalpy decreases when the contribution $\left(P v_{\mathrm{HB}}^{(\Phi)}-J_{\Phi}\right) N_{\mathrm{HB}}^{(\Phi)}$ decreases, i.e., when $N_{\mathrm{HB}}^{(\Phi)}$ increases. Therefore, we find that under depressurization the denaturation process is enthalpy driven.

From the Clapeyron relation $d P / d T=\Delta S / \Delta V$ applied to the SR [10], we expect that the $f \longleftrightarrow u$ process is isochoric at the SR turning points where $\partial T /\left.\partial P\right|_{\mathrm{SR}}=0$, while it is isoentropic at the turning points where $\partial P /\left.\partial T\right|_{\mathrm{SR}}=0$. In particular, at any $T$ and $P$ the volume change in the $f \rightarrow u$ process is given by

$$
\Delta V \equiv V_{u}-V_{f} \simeq v_{\mathrm{HB}}^{(b)} \Delta N_{\mathrm{HB}}^{(b)}+\left(v_{\mathrm{HB}, 0}^{(\Phi)}-k_{1} P\right) \Delta N_{\mathrm{HB}}^{(\Phi)} .
$$

We estimate Eq. (4) calculating the average volume $V_{u}$ and $V_{f}$ in a wide range of $T$ and $P$, equilibrating water around a completely unfolded protein state and a completely folded state (with $n_{\mathrm{rr}}=n_{\text {max }}$ ). Consistent with Hawley's theory [10], we find that the $T$ denaturation is accompanied by a positive entropy variation $\Delta S>0$ at high $T$ and an entropic penalty $\Delta S<0$ at low $T$, while the $P$ denaturation is accompanied by a decrease of volume $\Delta V<0$ at high $P$ and an increase of volume $\Delta V>0$ at low $P$ (Fig. 3). In particular, at $P=0.3\left(4 \epsilon / v_{0}\right)$, corresponding to $\approx 500 \mathrm{MPa}$, we find $\Delta V \approx-2.5 v_{0}$; hence, $|P \Delta V|=$ $0.75(4 \epsilon) \approx 17 \mathrm{~kJ} / \mathrm{mol}$, very close to the typical reported value of $15 \mathrm{~kJ} / \mathrm{mol}$ [7]. By varying the parameters $v_{\mathrm{HB}}^{(\Phi)}$ and $J_{\Phi}$ we find that the first is relevant for the $P$ denaturation, as expected because it dominates Eq. (3), while the second affects the stability range in $T$. Both combine in a nontrivial way to regulate the low- $T$ entropic penalty. We test our results including a small water- $\Phi$ residue attraction and find 


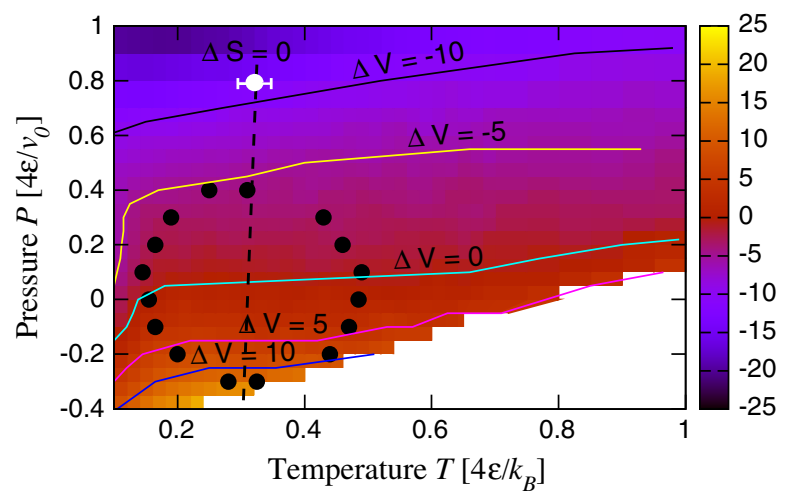

FIG. 3 (color online). Volume change $\Delta V$ for the $f \rightarrow u$ process in the $T-P$ plane. Color coded $\Delta V$ (black for negative, yellow for positive) is in $v_{0}$ units. Solid lines connect state points with constant $\Delta V$. Black points mark the SR. The locus $\Delta V=0$ has a positive slope and intersects the SR at the turning points with $d T /\left.d P\right|_{\mathrm{SR}}=0$. The dashed line, connecting the points with $d P /\left.d T\right|_{\mathrm{SR}}=0$, corresponds to the locus where $\Delta S=0$ and separates state points with $\Delta S>0$ (high $T$ ) from those with $\Delta S<0$ (low $T$ ) at the $f \rightarrow u$ process. The white symbol marks the error in the dashed-line slope estimate.

no qualitative differences but a small change in the $T$ range of the stability of the folded protein.

Next, we study the case of a protein with hydrophobic $(\Phi)$ and hydrophilic $(\zeta)$ residues $[12,67]$, with a residue-residue interaction matrix $A_{i, j}=\epsilon_{\mathrm{rr}}$ if residues $i$ and $j$ are $\mathrm{NN}$ in the unique native state, 0 otherwise. Water molecules interact with energy $\epsilon_{\mathrm{w}, \Phi}<J$ and $\epsilon_{\mathrm{w}, \zeta}>J$ with $\mathrm{NN} \Phi$ and $\zeta$ residues, respectively, accounting for the polarization of the solvent near the $\zeta$ residues. The polar $\zeta$ residues disrupt the tetrahedral order of the surrounding water molecules. Thus, we assume that a $\zeta$ residue $j$ and NN water molecules $i$ form a $\mathrm{HB}$ when the latter has $\sigma_{i, j}$ in the state $q_{j}^{(\zeta)}=1, \ldots, q$ preassigned to $j$. Finally, we consider that water-water enthalpy in the hydration shell is $H_{\lambda, \lambda} \equiv-J_{\lambda}+P v_{\mathrm{HB}}^{(\lambda)}$, if both molecules are NN to the same type of residue or $H_{\lambda, \mu} \equiv$ $\left(H_{\lambda, \lambda}+H_{\mu, \mu}\right) / 2$ if the NN residues belong to different types, with $\lambda, \mu=\Phi, \zeta$ and $J_{\zeta} \leq J$ [68] (Fig. 4).

Despite the complexity of the heteropolymer model, we find results that are similar to the homopolymer case, with the qualitative difference that with $\zeta$ residues we find a locus $\Delta V=0$ with negative slope and increased stability toward (i) cold and (ii) $P$ denaturation. In particular, for our specific choice of parameters, for the heteropolymer the cold denaturation at $P=0$ occurs below the glass transition, instead of $\approx 50 \%$ above as for the homopolymer. Furthermore, the SR against $P$ is $\approx 2$ times larger with $\zeta$ residues than without. This comparison suggests that the water contribution is relevant to the $f \longleftrightarrow u$ independently on the residue sequence, although the residue-residue interactions increase the stability of the folded state.

In conclusion, our model for protein folding reproduces the entire protein SR in explicit solvent and allows us to identify how water contributes to the $T$-and $P$-denaturation

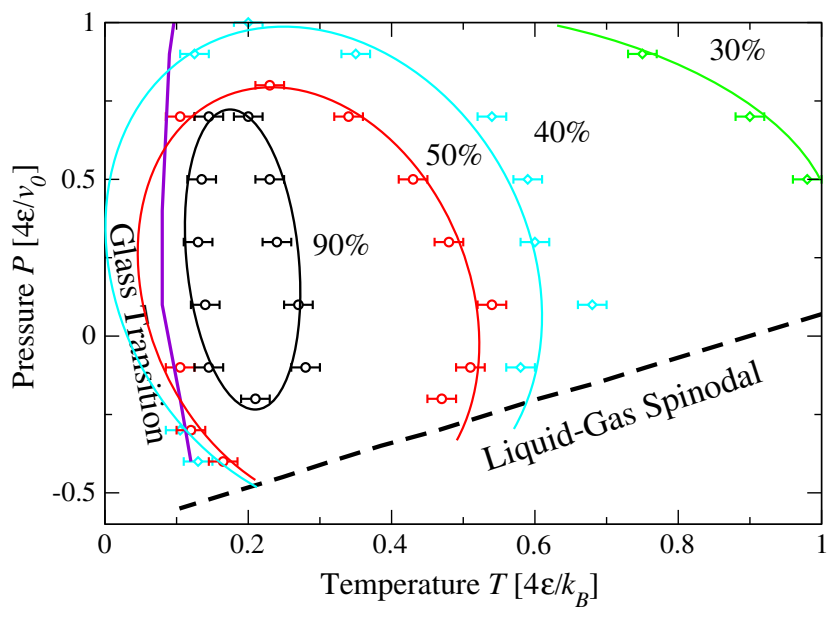

FIG. 4 (color online). The SR for the heteropolymer with a unique native state. We set $\epsilon_{\mathrm{rr}} / J=0.7, \epsilon_{\mathrm{w}, \Phi}=0, \epsilon_{\mathrm{w}, \zeta} / J=1.17$, $J_{\Phi} / J=1.3, J_{\zeta} / J=0.5, v_{\mathrm{HB}}^{(\zeta)}=0$, with all the other parameters as in Fig. 1. We test that changing the parameters, within physical ranges, modifies the SR, reproducing a variety of experimental SRs [11], but preserves the elliptic shape.

processes. The model is thermodynamically consistent with Hawleys theory but, in addition, allows for intermediate states for the $f \longleftrightarrow u$ process. We find that cold denaturation is energy driven, while unfolding by pressurization and depressurization, in addition to other suggested mechanisms [36], are density and enthalpy driven by water, respectively. For these mechanisms is essential to take into account how the protein-water interactions affect the stability of the water-water HB and the water density in the hydration shell. In particular, both properties control the low- $T$ entropic penalty. Our results are qualitatively robust against modification of the model parameters, within physical ranges, and the model is computationally efficient thanks to the adoption of a CG water model, representing a step towards the development of a theoretical and computational approach for protein design and engineering.

We thank M. Bernabei, I. Coluzza, C. Karner, E. Locatelli, P. Malgaretti and N. Patges for discussions, the Catalan FI-DGR 2010, the Italian Angelo della Riccia foundation, Spanish Ministerio de Economía y Competitividad FIS2012-31025, and EU NMP4-SL2011-266737 grants for support.

gfranzese@ub.edu

[1] Y. Levy and J. N. Onuchic, Annu. Rev. Biophys. Biomol. Struct. 35, 389 (2006).

[2] M. Kinoshita, Int. J. Mol. Sci. 10, 1064 (2009).

[3] R. Ravindra and R. Winter, ChemPhysChem 4, 359 (2003).

[4] A. Pastore, S. R. Martin, A. Politou, K. C. Kondapalli, T. Stemmler, and P. A. Temussi, J. Am. Chem. Soc. 129, 5374 (2007).

[5] F. Meersman et al., High Press. Res. An. Int. J. 19, 263 (2000). 
[6] G. Hummer, S. Garde, A. E. Garcia, M. E. Paulaitis, and L. R. Pratt, Proc. Natl. Acad. Sci. U.S.A. 95, 1552 (1998).

[7] F. Meersman, C. M. Dobson, and K. Heremans, Chem. Soc. Rev. 35, 908 (2006).

[8] N. V. Nucci, B. Fuglestad, E. A. Athanasoula, and A. J. Wand, Proc. Natl. Acad. Sci. U.S.A. 111, 13846 (2014).

[9] R. Wuttke, H. Hofmann, D. Nettels, M. B. Borgia, J. Mittal, R. B. Best, and B. Schuler, Proc. Natl. Acad. Sci. U.S.A. 111, 5213 (2014).

[10] S. A. Hawley, Biochem. 10, 2436 (1971).

[11] L. Smeller, Biochim. Biophys. Acta 1595, 11 (2002).

[12] P. De Los Rios and G. Caldarelli, Phys. Rev. E 62, 8449 (2000).

[13] M. I. Marqués, J. M. Borreguero, H. E. Stanley, and N. V. Dokholyan, Phys. Rev. Lett. 91, 138103 (2003).

[14] B. A. Patel, P. G. Debenedetti, F. H. Stillinger, and P. J. Rossky, Biophys. J. 93, 4116 (2007).

[15] C. L. Dias, T. Ala-Nissila, M. Karttunen, I. Vattulainen, and M. Grant, Phys. Rev. Lett. 100, 118101 (2008).

[16] C.-J. Tsai, J. V. Maizel, and R. Nussinov, Crit. Rev. Biochem. Mol. Biol. 37, 55 (2002).

[17] D. Paschek, S. Nonn, and A. Geiger, Phys. Chem. Chem. Phys. 7, 2780 (2005).

[18] M. V. Athawale, G. Goel, T. Ghosh, T. M. Truskett, and S. Garde, Proc. Natl. Acad. Sci. U.S.A. 104, 733 (2007).

[19] D. Nettels et al., Proc. Natl. Acad. Sci. U.S.A. 106, 20740 (2009).

[20] R. B. Best and J. Mittal, J. Phys. Chem. B 114, 14916 (2010).

[21] S. N. Jamadagni, C. Bosoy, and S. Garde, J. Phys. Chem. B 114, 13282 (2010).

[22] A. V. Badasyan, Sh. A. Tonoyan, Y. Sh. Mamasakhlisov, A. Giacometti, A. S. Benight, and V. F. Morozov, Phys. Rev. E 83, 051903 (2011).

[23] S. Matysiak, P. G. Debenedetti, and P. J. Rossky, J. Phys. Chem. B 116, 8095 (2012).

[24] D. Paschek and A. E. García, Phys. Rev. Lett. 93, 238105 (2004).

[25] D. Paschek, S. Gnanakaran, and A. E. Garcia, Proc. Natl. Acad. Sci. U.S.A. 102, 6765 (2005).

[26] T. Sumi and H. Sekino, Phys. Chem. Chem. Phys. 13, 15829 (2011)

[27] I. Coluzza, PLoS One 6, e20853 (2011).

[28] C. Dias, Phys. Rev. Lett. 109, 048104 (2012).

[29] P. Das and S. Matysiak, J. Phys. Chem. B 116, 5342 (2012).

[30] R. Sarma and S. Paul, Chem. Phys. 407, 115 (2012).

[31] A. Fernandez, J. Chem. Phys. 139, 085101 (2013).

[32] G. Franzese and V. Bianco, Food Biophys. 8, 153 (2013).

[33] S. Abeln, M. Vendruscolo, C. M. Dobson, D. Frenkel, and I. V. Baskakov, PLoS One 9, e85185 (2014).

[34] A. Ben-Naim, Eur. Phys. J. Spec. Top. 223, 927 (2014).

[35] C. Yang, S. Jang, and Y. Pak, Nat. Commun. 5, 5773 (2014).

[36] J. Roche, J. A. Caro, D. R. Norberto, P. Barthe, C. Roumestand, J. L. Schlessman, A. E. Garcia, B. GarciaMoreno E., and C. A. Royer, Proc. Natl. Acad. Sci. U.S.A. 109, 6945 (2012).

[37] B. J. Sirovetz et al., J. Phys. Chem. B 119, 11416 (2015).

[38] E. Larios and M. Gruebele, Methods 52, 51 (2010).

[39] H. W. Hatch, F. H. Stillinger, and P. G. Debenedetti, J. Phys. Chem. B 118, 7761 (2014).

[40] N. Muller, Acc. Chem. Res. 23, 23 (1990).

[41] G. Franzese, M. I. Marqués, and H. E. Stanley, Phys. Rev. E 67, 011103 (2003).
[42] K. Stokely, M. G. Mazza, H. E. Stanley, and G. Franzese, Proc. Natl. Acad. Sci. U.S.A. 107, 1301 (2010).

[43] M. G. Mazza, K. Stokely, S. E. Pagnotta, F. Bruni, H. E. Stanley, and G. Franzese, Proc. Natl. Acad. Sci. U.S.A. 108, 19873 (2011).

[44] F. de los Santos and G. Franzese, J. Phys. Chem. B 115, 14311 (2011).

[45] V. Bianco and G. Franzese, Sci. Rep. 4, 4440 (2014).

[46] Values $\epsilon=5.5 \mathrm{~kJ} / \mathrm{mol}, J / 4 \epsilon=0.5$, and $J_{\sigma} / 4 \epsilon=0.05$ give an average $\mathrm{HB}$ energy $E_{\mathrm{HB}} \simeq 23 \mathrm{~kJ} / \mathrm{mol}$ [42]. Here, to account for the ions in solution, we increase $5 \% \epsilon$ and decrease $J / J_{\sigma}$, resulting in a modification of the bulk phase diagram qualitatively similar to that induced by ions; see D. Corradini and P. Gallo, J. Chem. Phys. B 115, 14161 (2011). With our choice $\boldsymbol{\epsilon} \equiv 5.8 \mathrm{~kJ} / \mathrm{mol}, \boldsymbol{J} / 4 \boldsymbol{\epsilon}=0.3$ and $\boldsymbol{J}_{\sigma} / 4 \boldsymbol{\epsilon}=$ 0.05 we find $\boldsymbol{E}_{\mathrm{HB}} \simeq 20 \mathrm{~kJ} / \mathbf{m o l}$.

[47] L. Hernández de la Peña and P. G. Kusalik, J. Am. Chem. Soc. 127, 5246 (2005).

[48] A. K. Soper and M. A. Ricci, Phys. Rev. Lett. 84, 2881 (2000).

[49] C. Petersen, K.-J. Tielrooij, and H. J. Bakker, J. Chem. Phys. 130, 214511 (2009).

[50] Y. Tarasevich, Colloid J. 73, 257 (2011).

[51] J. G. Davis, K. P. Gierszal, P. Wang, and D. Ben-Amotz, Nature (London) 491, 582 (2012).

[52] S. Sarupria and S. Garde, Phys. Rev. Lett. 103, 037803 (2009).

[53] V. Bianco, S. Iskrov, and G. Franzese, J. Biol. Phys. 38, 27 (2012).

[54] K. Lum, D. Chandler, and J. D. Weeks, J. Phys. Chem. B 103, 4570 (1999).

[55] D. Schwendel, T. Hayashi, R. Dahint, A. Pertsin, M. Grunze, R. Steitz, and F. Schreiber, Langmuir 19, 2284 (2003).

[56] T. R. Jensen, M. Østergaard Jensen, N. Reitzel, K. Balashev, G. H. Peters, K. Kjaer, and T. Bjørnholm, Phys. Rev. Lett. 90, 086101 (2003).

[57] D. A. Doshi, E. B. Watkins, J. N. Israelachvili, and J. Majewski, Proc. Natl. Acad. Sci. U.S.A. 102, 9458 (2005).

[58] R. Godawat, S. N. Jamadagni, and S. Garde, Proc. Natl. Acad. Sci. U.S.A. 106, 15119 (2009).

[59] V. M. Dadarlat and C. B. Post, Biophys. J. 91, 4544 (2006).

[60] E. G. Strekalova, J. Luo, H. E. Stanley, G. Franzese, and S. V. Buldyrev, Phys. Rev. Lett. 109, 105701 (2012).

[61] T. Ghosh, A. E. García, and S. Garde, J. Am. Chem. Soc. 123, 10997 (2001).

[62] C. L. Dias and H.S. Chan, J. Phys. Chem. B 118, 7488 (2014).

[63] N. Giovambattista, P. J. Rossky, and P. G. Debenedetti, Phys. Rev. E 73, 041604 (2006).

[64] This approximation implies that our calculations are valid only for $P<1 / k_{1}$ where the HB contribution to the isothermal compressibility of the $\Phi$ hydration shell, $K_{T}^{(\mathrm{HB}, \Phi)} \equiv$ $-\left(1 / V^{(\Phi)}\right)\left(\partial V^{(\Phi)} / \partial P\right)_{T} \simeq\left[k_{1} /\left(1-k_{1} P\right)\right]-\left(1 / N_{\mathrm{HB}}^{(\Phi)}\right)\left(\partial N_{\mathrm{HB}}^{(\Phi)} /\right.$ $\partial P)_{T}$, is finite.

[65] Cavity contribution could cover water contribution to the $P$ denaturation $[7,36]$.

[66] S. V. Buldyrev, P. Kumar, P. G. Debenedetti, P. J. Rossky, and H. E. Stanley, Proc. Natl. Acad. Sci. U.S.A. 104, 20177 (2007).

[67] K. F. Lau and K. A. Dill, Macromolecules 22, 3986 (1989).

[68] J.-X. Cheng, S. Pautot, D. A. Weitz, and X. S. Xie, Proc. Natl. Acad. Sci. U.S.A. 100, 9826 (2003). 